\title{
A validated miRNA signature for the diagnosis of osteoporosis related fractures using SVM algorithm classification
}

\author{
XIAOLIN TANG ${ }^{1}$, YINSHAN BAI ${ }^{2}$, ZHIMING ZHANG ${ }^{1}$ and JIANLIN LU ${ }^{1}$ \\ ${ }^{1}$ Department of Medical Science, Shunde Polytechnic, Foshan, Guangdong 528300; \\ ${ }^{2}$ Life Science and Engineering College, Foshan University, Foshan, Guangdong 528231, P.R. China
}

Received March 16, 2018; Accepted April 29, 2020

DOI: $10.3892 / \mathrm{etm} .2020 .8928$

\begin{abstract}
The aim of the present study was to develop a circulating microRNA expression signature for early prediction of osteoporotic fractures and to validate the results using Gene Expression Omnibus (GEO) datasets. The GSE70318 dataset was downloaded from GEO and used to build an osteoporotic fracture prediction model based on the receiver operating characteristic curve and support vector machine (SVM) classification index. The GSE74209 dataset was used as a validation dataset. Additionally, in vitro, alkaline phosphatase (ALP) activity was measured in the presence or absence of microRNA (miRNA/miR) treatments in human osteoblast cells. The expression of two selected genes was detected by western blotting. miR-188-3p, miR-942-3p, miR-576-3p and miR-135a-5p were differentially expressed between controls and osteoporotic patients with fractures. SVM classification using these four miRNAs provided better dichotomization. It was further confirmed that miR-576-3p and 135a-5p in the GSE74209 dataset could also significantly discriminate between the controls and fracture patients, the area under the curve of SVM2 was 0.9722 with $95 \%$ CI $0.8885-1.056$. Further analysis indicated that the target genes of the two miRNAs participated in the Wingless-related integration site, Hedgehog and transforming growth factor- $\beta$ signaling pathways and osteoclast differentiation. miR-576-3p and miR-135-5p transfection decreased ALP activity and ALP activity was increased in the presence of blocking antisense oligonucleotides. Western blotting indicated miR-576-3p and miR-135-5p decreased CSNK1A1L and LRP6 levels, respectively. In conclusion, two miRNA signatures were developed and validated for the prediction of osteoporotic fractures.
\end{abstract}

Correspondence to: Dr Xiaolin Tang, Department of Medical Science, Shunde Polytechnic, 1st Medical Building, 2 East Desheng Road, Shunde, Foshan, Guangdong 528300, P.R. China E-mail: celinetangphd@163.com

Key words: osteoporosis, fracture, microRNA, receiver operating characteristic, support vector machine

\section{Introduction}

Osteoporosis is prevalent in the aging population and has become a major health threat worldwide. Osteoporotic fractures can seriously impact the quality of life in certain patient groups, for example, in post-menopausal women (1). Bone mineral density (BMD) and bone cell homeostasis are regarded as the two most important risk factors in fractures (2). Additionally, aberrant osteoblastic activities, such as depressed osteoblast maturation, and osteoclastic activities, such as resorption can significantly influence bone homeostasis. Fracture healing is a complex biological process that requires interactions amongst a series of different cell types and includes increasing differentiation and maturation of osteoblasts and osteoclast activity in bone remodeling (3).

MicroRNAs (miRNAs/miRs) have been reported as potential biomarkers of fracture prediction in osteoporotic patients (4). miRNAs negatively regulate gene expression through post-transcriptional mechanisms by endonuclease mediated target mRNA degradation. Numerous studies have revealed the importance of miRNAs in the regulation of bone formation, remodeling and homeostasis (5-7). An increasing quantity of miRNA array data is being uploaded and stored in the Gene Expression Omnibus (GEO) database. Using the GEO database, several studies have employed high throughput miRNA arrays to profile miRNA expression signatures for the purpose of biomarker identification $(8,9)$. Understanding of miRNA regulation and biomarker identification in bone metabolism has potential to significantly benefit miRNA-based therapeutics in bone disease. Seeliger et al (10), identified 6 miRNAs upregulated in osteoporotic fracture patients which included miR-21, miR-23a, miR-24, miR-25, miR-100 and miR-125b. Garmilla-Ezquerra et al (11), also detected miR-187 and miR-518f as being differentially expressed between the control and patients with osteoporotic fractures.

Whilst several studies have focused on miRNA expression levels in osteoporotic and healthy patients, cross validation in different cohorts remains challenging and is an under reported area (6). In osteoporotic derived fractures, studies have revealed varied miRNA expression profiles by comparing patients with fractures with healthy controls and certain reports have performed further validation $(12,13)$. The aim of the present study was to identify an aberrant miRNA expression signature in osteoporotic patients with recent fractures. 
miRNAs associated with osteoporotic fractures were identified and were validated across GEO datasets from different study cohorts. Furthermore, a support vector machine (SVM) algorithm-based miRNA expression signature model was built to predict the possibility of fractures in osteoporotic patients.

\section{Materials and methods}

Cell culture and reagents. Human hFOB1.19 cells (cat. no. CRL-11372) were purchased from the American Type Culture Collection. The culture medium for hFOB1.19 cells was a 1:1 mixture of Ham's F12 Medium and DMEM (Gibco; Thermo Fisher Scientific, Inc.), supplemented with $2.5 \mathrm{mM}$ L-glutamine (without phenol red; cat no. 25030081; Gibco; Thermo Fisher Scientific, Inc.), $0.3 \mathrm{mg} / \mathrm{ml} \mathrm{G} 418$ and $10 \% \mathrm{FBS}$. The cells were cultured in an incubator at $34^{\circ} \mathrm{C}$ with $100 \%$ humidity at $5 \% \mathrm{CO}_{2}$. Differentiation of hFOB1.19 was performed as described in a previous study (14). The sequences of miR-576-3p, miR-135a-5p, miR-576-3p and miR-135a-5p were as follows: 5'-AAGAUGUGGAAAAAUUGGAAUC-3', 5'-UAUGGCUUUUUAUUCCUAUGUGA-3', 5'-ATTCTA ATGGGCCACGTCTTT-3' and 5'-TATAGGGATACT TAGCCGTGGG-3' respectively. The miRNAs and anti-sense miRNAs were synthesized and constructed into pLVX-shRNA2-EGFP-Puro plasmids (controls) by Youbio Technology Co., Ltd. using pLVX-shRNA2-miR-576-3p (pLVX-miR-576-3p), pLVX-shRNA2-miR-576-3p -antisense (pLVX-miR-576-3p-antisense), pLVX-shRNA2-miR-135a-5p (pLVX-miR-135a-5p), pLVX-shRNA2-miR-135a-5p- antisense (pLVX-miR-135a-5p-antisense) vectors. MiRNAs containing plasmids and controls $(1 \mu \mathrm{g} / \mathrm{ml})$ were transfected using JetPRIME transfection reagent (PloySciences Inc.), according to the manufacturer's protocol. At $24 \mathrm{~h}$ post-transfection, adherent cells or culture supernatant were ready for subsequent treatment or collected for further analysis. Plasmid transfection efficiency was observed using fluorescence microscopy (magnification, x100; MF43; Micro-shot Technology Co., Ltd) and images were captured by microscopy-computer system (MShot Image Analysis System; v1.0; Micro-Shot Technology Co., Ltd).

Cohort of patients and controls. All information and expression data of the patient cohorts were downloaded from Gene Expression Omnibus database of the National Center for Biotechnology Information (ncbi.nlm.nih.gov/gds/). In the current study, according to the GSE70318 dataset, healthy post-menopausal women without type 2 diabetes were selected as the control group $(\mathrm{n}=17)$ and post-menopausal women with osteoporotic fractures without type 2 diabetes were selected as the experimental group ( $\mathrm{n}=19)$ (15). The GSE74209 dataset was defined as validated data, with 6 healthy controls and 6 fracture patients (8). Data are presented in Table I.

GEO matrix data and data normalization. Osteoporotic fracture patients and controls derived from the miRNA matrix dataset, GSE70318 (15), were used for initial analysis. An additional matrix dataset was downloaded, GSE74209 (8), for the validation study. Patient information and serological indices of these datasets are listed in Table I.

Although the miRNA arrays were performed on different platforms and completed by different research teams, the data were normalized for analysis using the geometric mean of the endogenous controls. In the GEO74209 dataset, none of the participants had a history of metabolic or endocrine diseases, chronic renal failure, chronic liver disease, malignancy, Paget's disease of bone, malabsorption syndrome and did not receive hormone replacement therapy, anti-resorptive or anabolic agents, oral corticosteroids, anti-epileptic drugs, or treatment with lithium, heparin or warfarin (8). Patient miRNA data were obtained from GEO, which is open access, free and does not require ethical approval or patient consent. The validated and predicted target genes of these miRNAs were searched and collected using online software and database (diana.imis. athena-innovation.gr/DianaTools/index.php?r=tarbase/index).

ROC curve analysis. Receiver operating characteristic (ROC) curve analysis is a commonly used method to visualize the performance of a binary classifier and is widely used in biostatistics. For the evaluation of diagnostic biomarkers, the sensitivity and specificity are of importance to their applications. Several miRNA biomarker studies have used the ROC curve method $(16,17)$. In the present study, miRNA expressions levels in patients with fractures were compared with healthy controls. Following ROC curve analysis, the statistical significance and area under the curve were analyzed for the selected miRNAs and plotted with $95 \%$ confidence intervals (CI) using GraphPad Prism v5.0 (GraphPad Software, Inc.).

SVM algorithm for classification of selected miRNAs. An SVM classifier algorithm was applied to determine the feasibility of fracture prediction using miRNA expression signatures in osteoporotic patients. The SVM classification algorithm was finished using MultiExperiment Viewer software (v4.2; GitHub Inc.) (18). The algorithm mechanism was completely studied and reported by Maulik and Chakraborty (19). The SVM algorithm is capable of reducing the misclassification rate of the miRNAs through a process of positive and negative filtering which results in increased accuracy. Briefly, the miRNA expression data were formatted into columns as raw data and saved as a .txt file. SVM classification function was chosen in the software menu bar. The miRNA expression data file was loaded for calculation. The output results showed the weight index of each miRNA. According to the SVM algorithm index, miRNA subsets from the training cohort were selected and combined, parameters were identified for each of the included miRNAs and the different SVM-based miRNA sets were compared (after calculation) using ROC curves. A higher value of area under the ROC curve represented a more accurate SVM classification. Various miRNA combinations were used until the highest value for area under the ROC curve was obtained for fracture prediction, suggesting the highest sensitivity and specificity for the biomarker signature.

Osteoblast alkaline phosphatase (ALP) activity. ALP activity of human cultured osteoblasts was examined using an ALP activity detection kit (cat. no. P0321; Beyotime Institute of Biotechnology, Inc.). Following stimulation by $10 \mathrm{ng} / \mathrm{ml}$ of transforming growth factor (TGF)- $\beta 1$ and $4 \mathrm{mM} \mathrm{L-glutamine} \mathrm{for} 3$ days, osteoblasts were treated with miRNA or miRNA antagonist for 2 days following pre-induction with TGF- $\beta 1$ and L-glutamine. ALP activity was determined in the cell culture supernatants and 
Table I. Summary of the patients and profile data from the GSE70318 and GSE74209 datasets.

\begin{tabular}{|c|c|c|c|c|}
\hline \multirow[b]{2}{*}{ Index } & \multicolumn{2}{|c|}{ GSE70318 } & \multicolumn{2}{|c|}{ GSE74209 } \\
\hline & Control & Fracture & Control & Fracture \\
\hline Number of patients & 17 & 19 & 6 & 6 \\
\hline Age (years) & $58.10 \pm 5.00$ & $64.70 \pm 5.80$ & $72.50 \pm 7.42$ & $75.16 \pm 3.54$ \\
\hline Height (cm) & $161.10 \pm 5.80$ & $162.40 \pm 8.20$ & - & - \\
\hline Weight (kg) & $68.00 \pm 13.70$ & $67.20 \pm 10.40$ & - & - \\
\hline Body mass index $\left(\mathrm{kg} / \mathrm{m}^{2}\right)$ & $26.00 \pm 4.40$ & $25.50 \pm 3.40$ & $26.06 \pm 3.25$ & $24.38 \pm 2.83$ \\
\hline Vitamin D $(\mu \mathrm{g} / \mathrm{l})$ & $27.70 \pm 12.10$ & $42.20 \pm 11.60$ & - & - \\
\hline Parathyroid hormone $(\mu \mathrm{g} / \mathrm{l})$ & $39.90 \pm 13.50$ & $32.30 \pm 23.90$ & - & - \\
\hline Bone mineral density $\left(\mathrm{g} / \mathrm{cm}^{2}\right)$ & $0.88 \pm 0.13$ & $0.83 \pm 0.07$ & $0.79 \pm 0.07$ & - \\
\hline Sample source & Serum & Serum & Bone tissue & Bone tissue \\
\hline
\end{tabular}

Data are shown as mean \pm standard deviation.

osteoblasts lysates, according to the manufacturer's protocol. All standards, test samples and buffers were pre-warmed to room temperature. A total of $50 \mu \mathrm{l} /$ well of p-nitrophenol as a substrate was added to a 96-well plate and $100 \mu \mathrm{l}$ of standard or unknown samples were mixed well with the substrate. The 96-well plate was incubated at $37^{\circ} \mathrm{C}$ in an incubator for $10 \mathrm{~min}$ and the reaction was stopped by adding $100 \mu 1$ of stop solution. The plate was read using a plate reader at a wavelength of $405 \mathrm{~nm}$ (BioTek Synergy 2; BioTek Instruments). ALP activity of the unknown samples was calculated according to the standard sample activity divided by the reaction time of $10 \mathrm{~min}$.

Western blotting. Protein expression levels were semi-quantified by western blotting as described previously (14). Osteoblasts were washed with ice-cold PBS 3 times before being lysed with ice-cold lysis buffer supplemented with protease and phosphatase inhibitors. Cell supernatant was collected after centrifugation at $12,000 \mathrm{x} \mathrm{g}$ at $4^{\circ} \mathrm{C}$ for $15 \mathrm{~min}$. A total of $\sim 20 \mu \mathrm{g} /$ lane samples were loaded onto a $10 \%$ SDS gel and resolved using SDS-PAGE. Proteins were transferred to Hybond $\mathrm{C}$ nitrocellulose membrane using a blotting cassette at a $100 \mathrm{v}$ in ice-cold conditions for $60 \mathrm{~min}$. The membrane was blocked with $5 \%$ non-fat milk in TBS-Tween (TBST) at room temperature for $30 \mathrm{~min}$. After blocking, the membrane was incubated with primary antibodies overnight at $4^{\circ} \mathrm{C}$ with slight shaking. A dilution of 1:2,000 in TBST was used for all primary antibody dilution. The primary antibody used were as follows: CSNK1A1L (cat. no. 2655; Cell Signaling Technology, Inc.), LRP6 (cat. no. 2560; Cell Signaling Technology, Inc.), $\beta$-catenin (cat. no. 8480; Cell Signaling Technology, Inc.) and actin (cat. no. sc-376421; Santa Cruz Biotechnology, Inc.). After washing four times in TBST, the membrane was probed with horseradish peroxidase-conjugated secondary antibodies (cat. nos. 111035003 and 115035062; Jackson ImmunoResearch Inc.; 1:3,000 in TBST) for a further $1 \mathrm{~h}$ at room temperature. Signals were visualized using ECL reagent (GE Healthcare) and detected using the ChemiDoc XRS gel documentation system (Bio-Rad Laboratories, Inc.).

Statistical analysis. In the initial miRNA data analysis, comparison between fracture and healthy control patients was tested using a Mann-Whitey U-test where $\mathrm{P}<0.05$ was considered to indicate a statistically significant difference. One-way ANOVA was performed for comparison of multiple groups and the Bonferroni test was used for post-hoc analysis. $\mathrm{P}<0.05$ was considered to indicate a statistically significant difference. In KEGG and GO annotation analyses, in order to reduce type I errors, a false discovery rate value of $<0.05$ and $\mathrm{P}<0.05$ was considered to indicate a statistically significant difference. All statistical analysis were performed using SPSS v13.0 (SPSS, Inc.) unless specifically indicated otherwise in the study.

\section{Results}

Aberrant expression of miRNAs in osteoporotic associated fractures. The GSE70318 dataset was downloaded from the GEO database which contained the expression levels of 153 miRNAs from healthy patients and patients with osteoporotic fractures. Quantification of serum circulating miRNAs was performed in a custom 384 well panel. Aberrant expression of circulating miRNAs was quantified as the fold change. To explore the potential for identification of diagnostic biomarkers, the sensitivity and specificity were calculated using ROC curves. Before performing ROC curve analysis, scatter dot plots were generated as shown in Fig. 1. The results showed that the expression of miR-188-3p, miR-942-3p, miR-181a-3p, miR-576-3p and miR-135a-5p were significantly different from the healthy controls $(\mathrm{P}<0.01$; Fig. 1A). In the ROC curve and AUC analysis, it was shown that miR-188-3p, miR-942-3p, miR-576-3p and miR-135a-5p had higher sensitivity (Fig. 1B) and the AUC values were: miR-188-3p, 0.889; miR-576-3p, 0.751; miR-135a-5p, 0.759; miR-942-3p, 0.678 miR-181a-3p, 0.817; and miR-330-3p, 0.342.

Female fracture risk factors using ROC curves and the SVM algorithm. For improved classification of patients with osteoporotic fractures from healthy controls, hierarchical clustering was used for dichotomization. A total of 6 miRNAs were identified which could accurately discriminate between fracture patients and healthy controls (Fig. 2A) with an accuracy of $>85 \%$ (16/19 cases). Furthermore, differentially expressed 

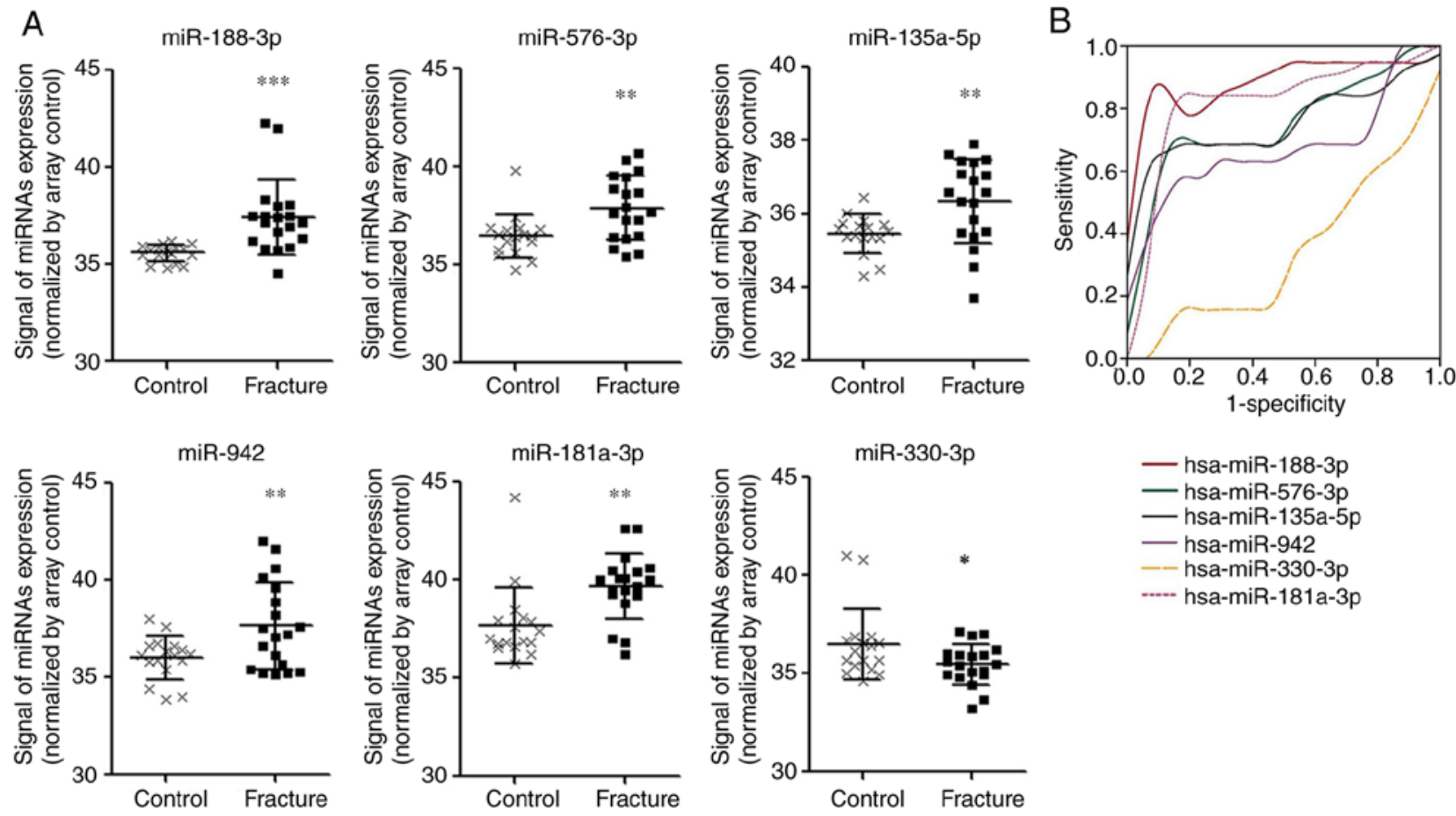

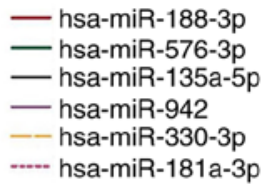

Figure 1. Aberrant circulating miRNA expression in the GSE70318 dataset. (A) miR-188-3p, miR-942-3p, miR-181a-3p, miR-576-3p and miR-135a-5p showed significant differences in their expression levels compared with the healthy controls. (B) Area under curve values of miR-188-3p, miR-181a-3p, miR-181a-3p and miR-576-3p showed significant sensitivity and specificity for classification of the controls and patient. ${ }^{*} \mathrm{P}<0.05 .{ }^{* * *} \mathrm{P}<0.01 .{ }^{* * * *} \mathrm{P}<0.001$. miRNA/miR, microRNA.

\section{A}

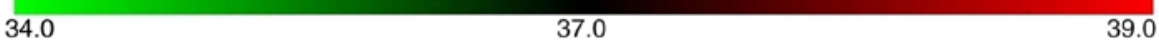

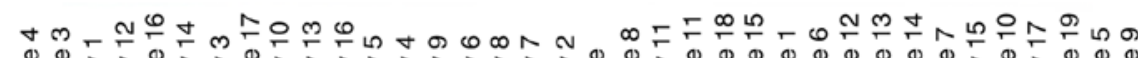

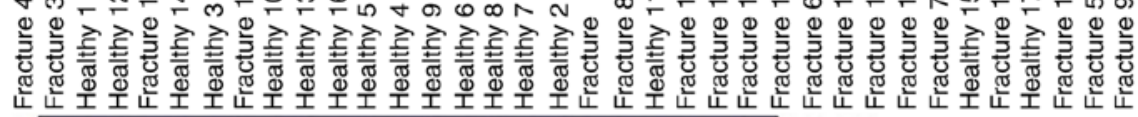

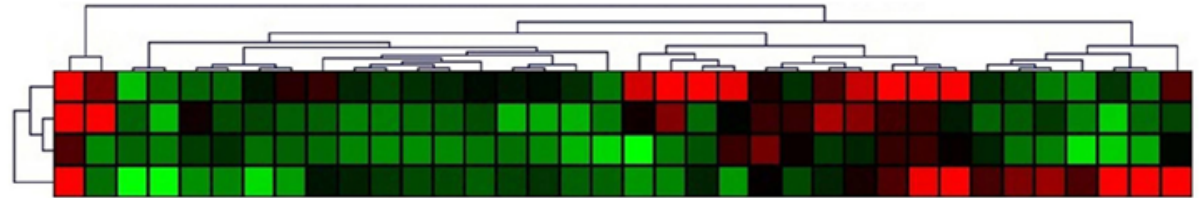

hsa-miR-576-3p hsa-miR-188-3p hsa-miR-135a-5p hsa-miR-942

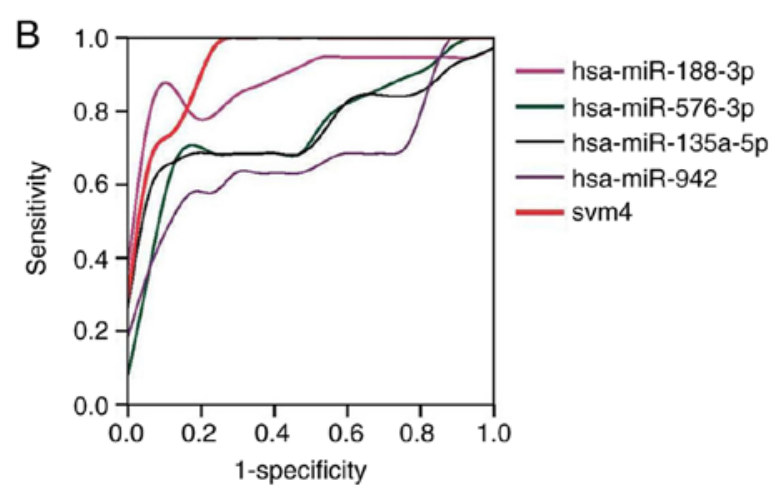

Figure 2. AUC value and SVM-based algorithm classification for fracture prediction. (A) Hierarchical clustering analysis for dichotomization of healthy controls and patients with osteoporotic fractures. (B) Accuracy of clustering was $>85 \%$ (16/19). SVM of four miRNAs showed the highest AUC (0.941) in all single miRNAs. AUC, area under the curve; SVM, support vector machine; miRNA/miR, microRNA.

miRNAs were clustered together to improve diagnostic confidence. miR-188-3p, miR-942-3p, miR-576-3p and miR-135a-5p were further calculated using the SVM classifier method in MultiView version 4.2. After SVM weighting and calculation, the data was input into SPSS for ROC curve analysis.
The SPSS analysis showed SVM 4-miR had the highest AUC (0.941) in all miRNAs (Fig. 2B), with the following AUC values: miR-188-3p, 0.889; miR-576-3p, 0.751; miR-135a-5p, 0.759; miR-942-3p, 0.678; and svm4, 0.941. The other two miRNAs, miR-181a-3p and miR-330-3p, did not show improved accuracy 

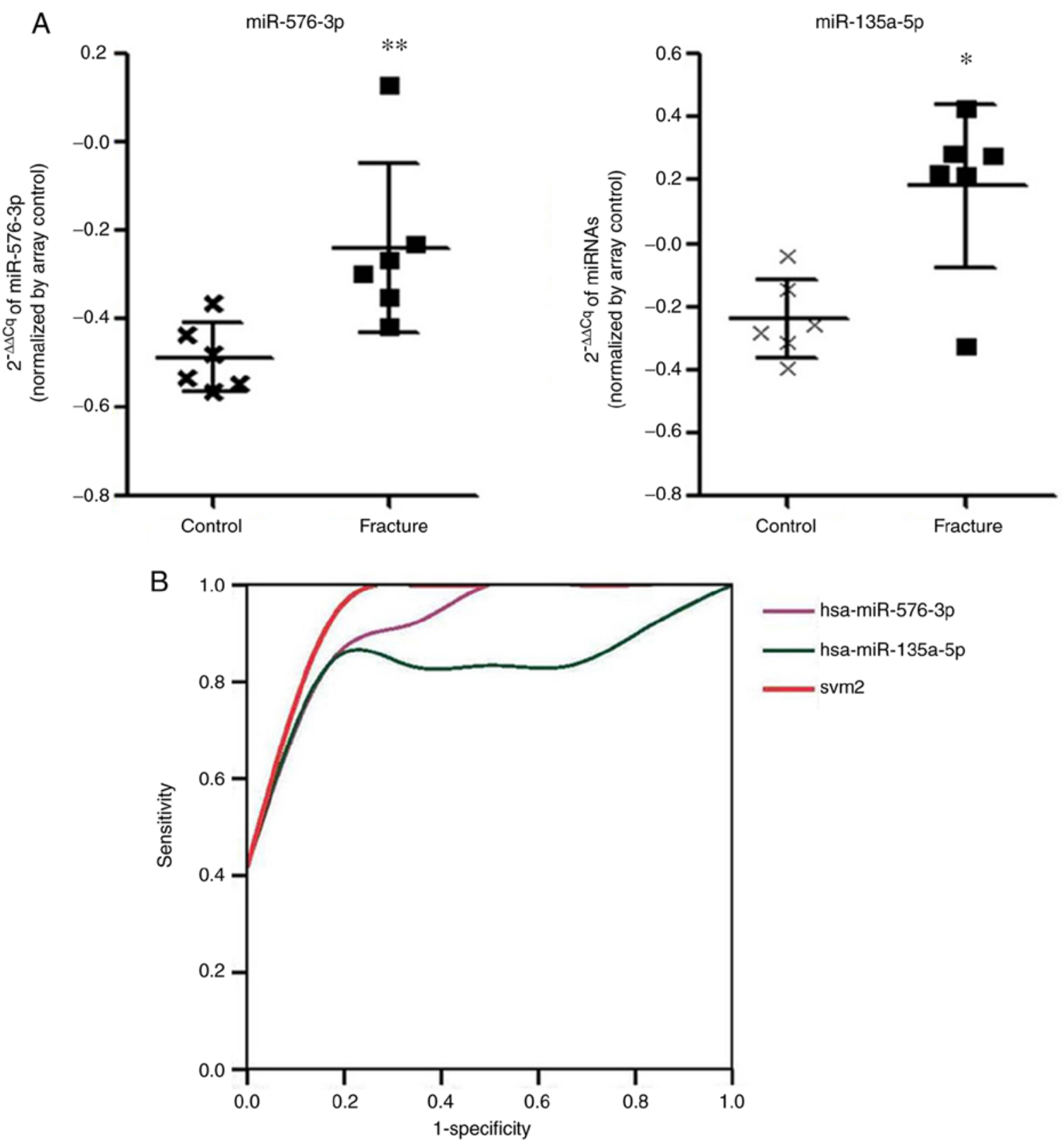

Figure 3. GSE72409 dataset validation. Statistical analysis showed that miR-576-3p and miR-135a-5p discriminate between patients with and without fractures. (A) AUCs were 0.9440 with a $95 \%$ CI of $0.8485-1.1584$ for miR-576-3p and 0.8611 with a 95\% CI of 0.6039-1.1119 for miR-135a-5p $\left({ }^{*} \mathrm{P}<0.05,{ }^{* *} \mathrm{P}<0.01\right)$. (B) SVM2 validation showed the AUC 0.9722 with $95 \%$ CI 0.8885-1.056. miRNA/miR, microRNA; CI, confidence interval; AUC, area under the curve.

in clustering with other miRNAs, which decreased accuracy to $78 \%$ and $75 \%$, respectively. Therefore, these two miRNAs were removed from the clustering of miRNAs. Collectively, the results indicated the vital importance of circulating miRNAs in the prediction of osteoporotic fractures. However, the use of miRNAs as biomarkers was still unreliable due to outcome data for patients with fractures not being available before and after fractures. In addition, the findings require cross study validation using different patient cohorts with different demographics and disease-related variables in both controls and fracture patients.

Cross validation with a different GEO dataset. To confirm the above findings, the miRNA expression signature was validated in another GEO dataset, GSE74209. The experimental design and the included patients fitted the above training cohort (GSE70318) precisely. Patient information and serological indices of these datasets are listed in Table I. After comparison, it was confirmed that miR-576-3p and miR-135a-5p could discriminate between osteoporotic patients with and without fractures with an AUC of 0.9440 and $95 \%$ CI of 0.8485-1.1584 and 0.8611 with $95 \%$ CI of 0.6039-1.1119, respectively (Fig. 3A). SVM2 classification consisting of the above two miRNAs showed the highest AUC, with values of 0.972 with $95 \%$ CI of 0.8885-1.230 (Fig. 3B), the AUC of miRNAs were: miR-576-3p, 0.944 ; miR-135a-5p, 0.861; and svm2, 0.972. There was no statistically significant difference in miR-188 and miR-942 in this dataset (data not shown) and it was not possible to perform further validation as other datasets were not available. 
Two miRNA signature in KEGG and GO annotation analysis. The function of miRNAs is mediated primarily through transcriptional modification by RNA-induced silencing complex (RISC). RISC cuts the miRNA-mRNA complex and degrades the mRNA (20). The validated and predicted target genes of these miRNAs were searched and collected using online software and database (diana.imis.athena-innovation. gr/DianaTools/index.php?r=tarbase/index).

KEGG signal pathway and GO annotation analysis were used to analyze the selected miRNAs that were differentially expressed in osteoporotic fractures (data not shown). When combined, the analysis of KEGG and GO annotation, the most significantly associated pathways were associated with cancer as they were tightly linked to cellular metabolism, dead cell clearance, organ development, cell growth and ubiquitination. Although KEGG signaling pathway analysis is usually employed to elucidate associated pathways containing differentially expressed genes, the value of the KEGG pathway may be negative even if the input gene served a vital role in the pathway. Due to the small number of input gene numbers and the GO annotation analysis, it was not possible to delineate meaningful pathways, GO functions or GO components in bone formation. In order to identify which pathways were involved in osteoporotic fractures associated with the regulation of the 4-miRNA signature, bone formation, metabolism and homeostasis associated with signaling pathways were analyzed. Bone formation associated genes and signaling pathways were reported in bone biology and bone signaling pathways (21). The Wingless-related integration site (WNT), Hedgehog, TGF- $\beta$ and osteoclast differentiation pathways were selected for further analysis based on previous studies on bone biology (22-24). Several genes which were regulated by miR-57-3p and miR-135a-3p co-participated in the aforementioned pathways (Table II). The results of the present study showed that several genes were involved in bone formation and homeostasis including WNT3/4, CSNK1A1L, TNFRSF11B, LRP6 and PTCH1.

miR-576-3p and miR-135a-5p stimulates differentiation of human osteoblasts and mineralization through the WNT signaling pathway. It was hypothesized that the increased levels of miR-188-3a, miR-576-3p, miR-942-3p and miR-135a-5p in serum and bone cells may downregulate the expression of bone formation genes and therefore cause fractures. To determine if the miR-576-3p and miR-135a-3p could steadily regulate bone formation, a miRNA sequence was constructed into expression vectors and osteoblasts were transfected in vitro. The transfection efficiency of miRNA or anti-sense miRNA containing plasmids are shown in Fig. S1. In the cellular function experiments, bone formation biomarkers were used to determine the effects of miR-576-3p and miR-135-5p blocking antisense oligonucleotides. In the ALP activity assay, results showed that transfection of miR-576-3p or miR-135-5p decreased ALP activity $(\mathrm{P}<0.05$; Fig. $4 \mathrm{~A})$. The effects were further confirmed at the protein level by measuring expression of CSNK1A1L and LRP6 via western blotting. The data showed that miR-576-3p and miR-135-5p decreased CSNK1A1L and LRP6 protein levels, respectively (Fig. 4B and C). In the combination treatment group, the two miRNAs significantly decreased $\beta$-catenin expression. 

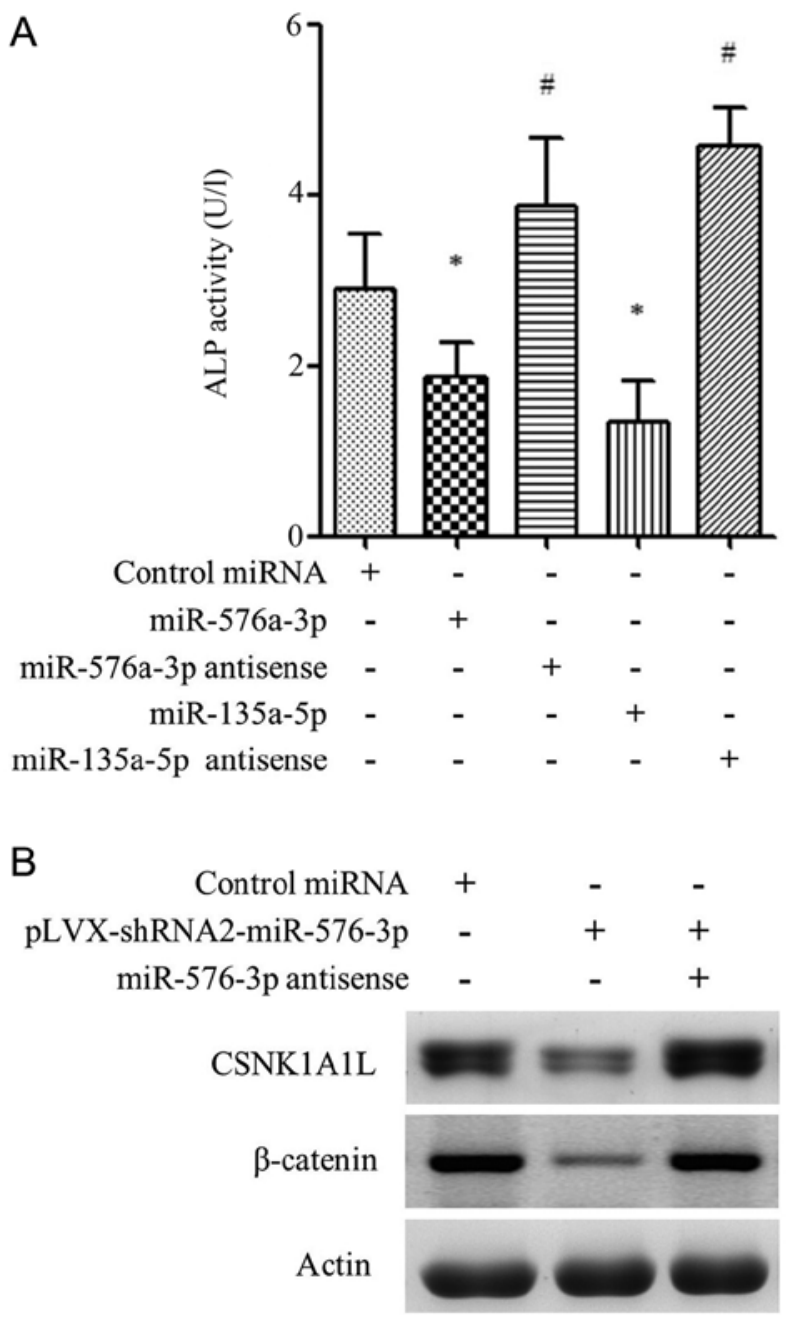
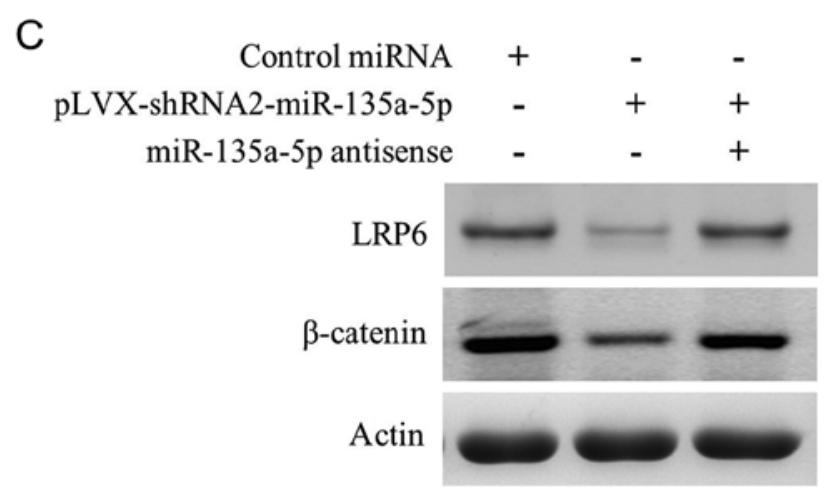

Figure 4. ALP activity and miRNA target validation. (A) miR-576-3p and miR-135-5p transfection decreased ALP activity, whilst transfection of the respective antisense sequences increased ALP activity. (B) miR-576-3p transfection decreased CSNK1A1L protein expression levels and antisense miR-576-3p reversed the decrease in expression levels to normal levels, as well as the levels of $\beta$-catenin. (C) miR-135a-5p decreased LRP6 and $\beta$-catenin protein levels and transfection of the antisense nucleotide reversed the decrease in protein expression levels. ${ }^{*} \mathrm{P}<0.05$ with controls. ${ }^{\text {}} \mathrm{P}<0.01$ compared with the miR-576-3p or miR135a-3p groups. ALP, alkaline phosphatase; miRNA/miR, microRNA.

\section{Discussion}

Analysis of the GSE70318 dataset comparing osteoporotic fracture patients and healthy controls was combined with significantly expressed miRNAs using the SVM classifier method. The results indicated that the SVM index from a 4-miRNA expression signature showed the highest AUC (0.941) in all miRNAs. Subsequently, the findings in different GEO datasets were validated and the SVM classification algorithm method was applied in the cross validation analysis. It was confirmed that miR-188-3p and miR-942-3p expression was higher in patients with high BMD compared with low BMD (data not shown). miR-576-3p and miR-135a-5p were shown to exhibit important roles in the development of osteoporosis as shown in the GSE74209 dataset, indicating the vital importance of circulating miRNAs in the prediction of osteoporotic fractures.

Seeliger et al (10), identified 6 miRNAs that were upregulated in osteoporotic fracture patients including miR-21, miR-23a, miR-24, miR-25, miR-100 and miR-125b. Garmilla-Ezquerra et al (11), also showed miR-187 and
miR-518f were differentially expressed between controls and osteoporotic fracture cohorts. Comparison between serum circulating miRNA and fresh bone tissue from the fracture site showed it was difficult to differentiate the most appropriate sample for biomarker development. Fresh bone tissues from fractured sites provided a scenario closer to the pathophysiological situation. It was speculated that the pathophysiology of osteoporosis ultimately causing fractures had a heterogeneous etiology with different miRNA expression patterns (8). However, for the prediction of early fractures, circulating miRNAs may have significant utility. In the present study, a circulating miRNA signature was used to cross validate the miRNA signature from bone tissues and it was hypothesized that there were inter-connections between circulating miRNAs and fractured bone tissue. The primary factor may be closely associated with the bone weakening induced by osteoporosis, whilst external pressures may only exert a minor role in causing osteoporotic fractures. Thus, circulating miRNAs may better reflect the entire mechanism occurring in patients with osteoporotic fractures. Throughout the present study, a complete identical miRNA signature for osteoporotic fracture 
was not found. This was not only because of the complexity of the pathophysiology of osteoporosis and fractures, but also due to the lack of identical organization of patients and controls in the study. Therefore, patients with osteoporotic fractures were paired with healthy controls from a similar experimental platform, to improve the analysis and allow a true platform for cross validation. However, similar experimental designs and cohort criteria are required for improved data validation.

Both GSE60230 and GSE70318 cohorts employed post-menopausal patients suffering osteoporotic fractures. Therefore, cross validation was performed using GSE60230. However, an aberrantly expressed miRNA signature was not found as in the case of GSE70318. Another different specific miRNA signature was found, which has also been previously reported (15). Although the GSE60230 cohort fitted the GSE70318 cohort, the sample size $(n=7)$ was too small to be used.

In the cross validation with BMD associated aberrant miRNA expression, the GSE63446 dataset used high BMD or low BMD as a risk factor in post-menopausal osteoporosis. Validation results showed that miR-188-3p and miR-942-3p expression was increased in patients with high BMD compared with patients with low BMD (data not shown). A previous study also indicated that miR-188-3p serves an important role in osteoporosis (15). miR-942-3p participates in osteoporotic development through regulation of the $\mathrm{WNT} / \beta$-catenin pathway (25). IFN- $\gamma$ derived mesenchymal stem cells have also been shown to improve fracture healing (26). However, due to the limited number of patients $(n=5)$ in the aforementioned study, the dataset lacked statistical power. Other miRNAs which did not show any difference in the GSE63446 dataset may be attributed to the small sample size. Additionally, the GSE74209 dataset used bone tissues from patients with arthritis and patients with osteoporotic fractures to develop an miRNA signature (8). miR-576-3p and miR-135a-5p were validated for the diagnosis of osteoporotic fractures and for their feasibility of prediction. In addition to the findings from the GSE74209 dataset and the present study, other reports have revealed the important roles of these miRNAs in osteoporosis and fractures $(15,27,28)$.

In conclusion, the present study developed a novel circulating miRNA signature for prediction of osteoporotic fractures and validated these miRNAs in different GEO datasets. These miRNAs may serve as biomarkers in monitoring of patients with osteoporosis and for prediction of fracture risk to inform possible fracture prevention strategies.

\section{Acknowledgements}

The authors would like to thank Dr Caiguo Ye (Department of Pathophysiology, Guangdong Medical University, Dongguan, Guangdong 523000, P.R. China) for his help with the ROC and SVM algorithm methods.

\section{Funding}

The present study was supported by Foshan Bureau of Science and Technology grant (grant no. 2018AB001903) and the Guangdong Education platform of Science and Technology Grant Youth Talent Project (grant no. 2018GkQNCX027).

\section{Availability of data and materials}

All data generated or analyzed during this study are included in this published article.

\section{Authors' contributions}

XT designed the study, performed data analysis, finished the ALP assay experiments and wrote the manuscript. YB performed GEO database mining and analysis, the ROC and SVM algorithm calculations and revised the manuscript. ZZ carried out the cell cultures, transfections and western blotting, and offered experimental materials and facilities. JL designed the miR-576-3p microRNA and anti-sense RNA, conducted the transfection experiments and wrote the sections on miRNA and transfections in the manuscript. All authors read and approved the final manuscript.

\section{Ethics approval and consent to participate}

Not applicable.

\section{Patient consent for publication}

Not applicable.

\section{Competing interests}

The authors declare that they have no competing interests.

\section{References}

1. An T, Hao J, Sun S, Li R, Yang M, Cheng G and Zou M: Efficacy of statins for osteoporosis: a systematic review and meta-analysis. Osteoporos Int 28: 47-57, 2017.

2. McNabb BL, Vittinghoff E, Schwartz AV, Eastell R, Bauer DC, Ensrud K, Rosenberg E, Santora A, Barrett-Connor E and Black DM: BMD changes and predictors of increased bone loss in postmenopausal women after a 5-year course of alendronate. J Bone Miner Res 28: 1319-1327, 2013.

3. Sousa CP, Dias IR, Lopez-Peña M, Camassa JA, Lourenço PJ, Judas FM, Gomes ME and Reis RL: Bone turnover markers for early detection of fracture healing disturbances: A review of the scientific literature. An Acad Bras Cienc 87: 1049-1061, 2015.

4. Weilner S, Skalicky S, Salzer B, Keider V, Wagner M, Hildner F, Gabriel C, Dovjak P, Pietschmann P, Grillari-Voglauer R, et al: Differentially circulating miRNAs after recent osteoporotic fractures can influence osteogenic differentiation. Bone 79: 43-51, 2015.

5. Taipaleenmäki H, Bjerre Hokland L, Chen L, Kauppinen S and Kassem M: Mechanisms in endocrinology: micro-RNAs: targets for enhancing osteoblast differentiation and bone formation. Eur J Endocrinol 166: 359-371, 2012.

6. Suttamanatwong S: MicroRNAs in bone development and their diagnostic and therapeutic potentials in osteoporosis. Connect Tissue Res 58: 90-102, 2017.

7. Chen J, Qiu M, Dou C, Cao Z and Dong S: MicroRNAs in bone balance and osteoporosis. Drug Dev Res 76: 235-245, 2015.

8. De-Ugarte L, Yoskovitz G, Balcells S, Güerri-Fernández R, Martinez-Diaz S, Mellibovsky L, Urreizti R, Nogués X, Grinberg D, García-Giralt N, et al: MiRNA profiling of whole trabecular bone: Identification of osteoporosis-related changes in MiRNAs in human hip bones. BMC Med Genomics 8: 75, 2015.

9. Lei SF, Papasian CJ and Deng HW: Polymorphisms in predicted miRNA binding sites and osteoporosis. J Bone Miner Res 26: 72-78, 2011.

10. Seeliger C, Karpinski K, Haug AT, Vester H, Schmitt A, Bauer JS and van Griensven M: Five freely circulating miRNAs and bone tissue miRNAs are associated with osteoporotic fractures. J Bone Miner Res 29: 1718-1728, 2014. 
11. Garmilla-Ezquerra P, Sañudo C, Delgado-Calle J, Pérez-Nuñez MI, Sumillera M and Riancho JA: Analysis of the bone microR Nome in osteoporotic fractures. Calcif Tissue Int 96: 30-37, 2015.

12. Zhang Y, Gao Y, Cai L, Li F, Lou Y, Xu N, Kang Y and Yang H: MicroRNA-221 is involved in the regulation of osteoporosis through regulates RUNX2 protein expression and osteoblast differentiation. Am J Transl Res 9: 126-135, 2017.

13. Meng J, Zhang D, Pan N, Sun N, Wang Q, Fan J, Zhou P, Zhu W and Jiang L: Identification of miR-194-5p as a potential biomarker for postmenopausal osteoporosis. PeerJ 3: e971, 2015.

14. Tang X, Lin J, Wang G and Lu J: MicroRNA-433-3p promotes osteoblast differentiation through targeting DKK1 expression. PLoS One 12: e0179860, 2017.

15. Heilmeier U, Hackl M, Skalicky S, Weilner S, Schroeder F, Vierlinger K, Patsch JM, Baum T, Oberbauer E, Lobach I, et al: Serum miRNA signatures are indicative of skeletal fractures in postmenopausal women with and without type 2 diabetes and influence osteogenic and adipogenic differentiation of adipose tissue-derived mesenchymal stem cells in vitro. J Bone Miner Res 31: 2173-2192, 2016.

16. Lu M, Kong X, Wang H, Huang G, Ye C and He Z: A novel microRNAs expression signature for hepatocellular carcinoma diagnosis and prognosis. Oncotarget 8: 8775-8784, 2017.

17. Zhou X, Zhu W, Li H, Wen W, Cheng W, Wang F, Wu Y, Qi L, Fan Y, Chen Y, et al: Diagnostic value of a plasma microRNA signature in gastric cancer: A microRNA expression analysis. Sci Rep 5: 11251, 2015.

18. Saeed AI, Sharov V, White J, Li J, Liang W, Bhagabati N, Braisted J, Klapa M, Currier T, Thiagarajan M, et al: TM4: A free, open-source system for microarray data management and analysis. Biotechniques 34: 374-378, 2003.

19. Maulik U and Chakraborty D: Fuzzy preference based feature selection and semisupervised SVM for cancer classification. IEEE Trans Nanobioscience 13: 152-160, 2014.
20. Bernstein E, Caudy AA, Hammond SM and Hannon GJ: Role for a bidentate ribonuclease in the initiation step of RNA interference. Nature 409: 363-366, 2001.

21. Iñiguez-Ariza NM and Clarke BL: Bone biology, signaling pathways, and therapeutic targets for osteoporosis. Maturitas 82: 245-255, 2015 .

22. Luo M, Huang HX, Huang H, Li ZT and Lai YY: Hedgehog signaling pathway and osteoporosis. Zhongguo Gu Shang 27: 169-172, 2014 (In Chinese).

23. Regan JN, Trivedi T, Guise TA and Waning DL: The role of TGF 3 in bone-muscle crosstalk. Curr Osteoporos Rep 15: 18-23, 2017.

24. Saad FA: Novel insights into the complex architecture of osteoporosis molecular genetics. Ann N Y Acad Sci 1462: 37-52, 2019.

25. McCubreyJA,FitzgeraldTL,YangLV,LertpiriyapongK,SteelmanLS, Abrams SL, Montalto G, Cervello M, Neri LM, Cocco L, et al: Roles of GSK-3 and microRNAs on epithelial mesenchymal transition and cancer stem cells. Oncotarget 8: 14221-14250, 2017.

26. Chi Y, Cui J, Wang Y, Du W, Chen F, Li Z, Ma F, Song B, $\mathrm{Xu} \mathrm{F}$, Zhao Q, et al: Interferon- $\gamma$ alters the microRNA profile of umbilical cord-derived mesenchymal stem cells. Mol Med Rep 14: 4187-4197, 2016.

27. Fang S, Deng Y, Gu P and Fan X: MicroRNAs regulate bone development and regeneration. Int J Mol Sci 16: 8227-8253, 2015.

28. Panizo S, Naves-Díaz M, Carrillo-López N, Martínez-Arias L, Fernández-Martín JL, Ruiz-Torres MP, Cannata-Andía JB and Rodríguez I: MicroRNAs 29b, 133b, and 211 regulate vascular smooth muscle calcification mediated by high phosphorus. J Am Soc Nephrol 27: 824-834, 2016.

This work is licensed under a Creative Commons Attribution-NonCommercial-NoDerivatives 4.0 International (CC BY-NC-ND 4.0) License. 